\title{
A Methodology to Develop a Mobile Application Model to Appraise Housing Design Quality
}

\author{
https://doi.org/10.3991/ijim.v11i6.6379 \\ Afaq Hyder Chohan \\ Ajman University of Science and Technology, Ajman, UAE \\ Haryanti Mohd Affandi \\ Universiti Kebangsaan Malaysia, Bangi, Selangor, MALAYSIA \\ Jihad Awad \\ Ajman University of Science and Technology, Ajman, UAE \\ Adi Irfan Che-Ani ${ }^{(凶)}$ \\ Universiti Kebangsaan Malaysia, Bangi, Selangor, MALAYSIA \\ adiirfan@gmail.com
}

\begin{abstract}
The variety of planning and design solutions occasionally turns into an underpinning factor for the defects and maintenance issues in built forms. Defects and maintenance that emerges from deficient design indicate that the design has not been conceded in the perception of quality. Reviewing the nature of deficient designs and resulting maintenance problems in various types of built forms revealed that housing sector is most effective of this phenomenon. The reason could be set as; housing planning, design, and construction are a most practiced activity to address the living needs of the ever-growing world population. This research considered that design quality of housing is severely affected in developing countries. In this regard housing standards and bylaws plays a minor role to ensure the housing quality in developing regions. However, it is notable that, in developing regions, the majority of populations have easy access to mobile communication technology. Therefore, it is possible to use mobile technology to educate the people about quality issues of housing and to help them to evaluate the quality of proposed or existing housing. At present, the use of mobile technology has not only heightened the need for IT knowledge but also extend the capabilities of many designers/architects and builders. Hence, there is a niche to develop an integrated hub that could appraise the function of each professional involved in housing processes. This research has established a criterion that, the mobile technology can help common people to understand the quality of house/housing and to ascertain it. The results of the research have surfaced a model of mobile application that could be used later to prepare the real-time mobile application to work as a "design quality assessment tool."
\end{abstract}

Keywords-Design Quality, Housing Quality, Information Technology, Mobile Application. 


\section{Introduction}

The life of an individual and family unfolds in the space within a house, formulating such a space and its standards should start from recognizing the quality of space that has to be provided in the family house to satisfy these needs. There is a general acknowledgement that enabling the market has failed to provide affordable, adequate housing for the predominant low-income households in the rapidly urbanizing parts of the world [2]. In fact, developing countries striving hard to prevent themselves from being swallowed by the big economies and this trend of developing nations results in unplanned growth, development and law transient in almost all walks of life particularly in urban centers. Also, the alarming rate of population growth is another problem faced by these countries, which ultimately creates the demand for increased number of housing facilities.

In developing countries, housing has remained subject to research and discussion on some reputable platforms. United Nations (2014) mentioned that the world had crossed a demographic milestone, in $2014,54 \%$ of the global population lived in urban areas, up from $34 \%$ in 1960 . Urban population growth is expected to continue and the UN estimates that by $2050,66 \%$ of the global population will live in cities. 2 This growth is predominantly occurring in Asia and Africa in countries that are often unprepared and unable to meet the growing needs of urban residents. Moreover, rapid urbanization resulting housing problems and it is most dominant phenomenon in all developing countries and it is inevitable since it is directly linked with their economic growth [1]. However, facts indicated that urban population forms an overwhelming majority in all developed countries. UN-Habitat (2016) also supported the finding and stated that housing and its quality in developing nations is at stake [3]. Moreover, World Bank (2014) notifies that larger fraction of the population living in developing world has little access to quality housing [2]. Perhaps it is suggested in some reports that, developing countries are severely affected by housing issue because they are not only facing housing debts but also quality housing for their citizens is emerging issue of developing countries.

The importance of design quality is articulated by the report published by Commission for Architecture \& Built Environment [21]. The report has highlighted the importance of design quality in buildings and warned the people about possible repercussions of poor design. The report declares that absence of design quality is likely to have significant adverse environmental, social and economic effects. Furthermore, the report adds that continuation of low design quality can lower the quality of life. Both the discussions above support the importance of design quality and notify the common men about the unexpected situation related to the low design quality of the building. These studies further declared that habitats of the low-quality building could face various types of jeopardy ranging from environmental, social and financial risks. Moreover, the study clearly suggests the major role of design quality in the housing provision, the absence of which results in unfortunate implications.

The discussion above about housing in developing countries and its quality is aligned with various aspect of building design and can be summarized as; the private sector is largely responsible for providing housing in developing countries, and its 
existing state suggests that it is an important issue that needs further research. The matter needs more brainstorming for improvement of housing design and quality construction in developing countries. The discussion also reveals that the end users (population) of developing countries are experiencing issues of quality housing in their daily life, and there is a gap between their understandings of housing and its quality.

Moreover, in many countries, no formal housing and land procurement / administration system or framework is widely available. In fact, only 25 to 30 countries in the world have a system that provides nationwide coverage for land and housing procurement. Whereas, most developing countries have less than 30 percent coverage of a formal system [4]. The study has proposed an integrated management model to formalize the land and housing administration in developing countries, through a multi-partner software development initiative (Social Tenure Domain Model-STDM).

This research consider growing population and need of urban housing in developing countries intakes much resources (money, materials, land, energy and so forth.) but are always inadequate in compare to need. Therefore, productive use of such resources is possible by adopting an integrated management system to review the certain issues related to housing. This approach is essential for resource efficient and equitable solution to housing, its quality and other urban problems. The need of a potential problem-solving program capable to meets the specified criteria and promise the better design quality thus making the IT and computer use indispensable.

In present days, information technology (IT) based (mobile application platform) has proven its effectiveness and usability in various fields [12]. The people today are busy, and on the move, they are using mobile application platforms to get information and updates about various essential matters related to their daily life i.e. from road navigation to weather forecast. According to Cyber Gear (2016), mobile applications are increasingly playing a vital role in business with a larger customer base as well as workforce relying on the convenience of access on-demand to information and solutions [5]. The study stress that apps are becoming the dominant form of digital interaction because people are spending much of that digital time on smart mobile devices, time spent per day on mobile devices has increased $575 \%$ in three years and $80 \%$ of the time devoted to mobile devices is spent using apps.

Scott Shane (2016), mention that rise in mobile-phone usage means that smartphone apps have become a key tool for various businesses because mobile apps increase engagement with customers [6]. It is a well-acknowledged fact that IT-based learning platforms are paving the way for common people to learn very fast about issues related to their daily life. However, at present in the housing sector of developing countries, the common people or end user have limited or no access to ascertain the quality of available housing stock. Thus, the niche is present to develop an ITbased mobile application to ascertain the housing quality and to educate common people about issues of housing, in shortest possible time through exciting ways.

The mobile application could be considered as a medium for educating people about housing and its quality standards. Because the mobile application is capable of grasping the attention of people very fast and it is very easy to use. This study considers that by providing an understanding of such issues to common people in these re- 
gions could improve the housing and its quality. The need is to develop the reliable, fast and suitable information technology-based teaching platform to aware the people about issues of housing. Therefore, this paper discussed on the methodology of the mobile application model development in appraising housing design quality.

\section{Need of mobile application in housing sector}

Following the timeline of design monitoring (IT) systems in architecture reveals that various researchers have stressed the use and development of the IT-based system in architecture for quality assessment.

a) Jackson (1999) has concluded with three reasons to adopt the automation system [7], which are :

- The expertise of human is perishable. However, computer expertise is permanent.

- Human expertise is hard to transfer, whereas computer-based knowledge and expertise can be shared and transfer easily.

- Human expertise is very expensive.

b) Rivard et.al (1999), proposed a shared conceptual model for building envelope design process, to provide communication between different members of building design team [8].

c) Altunay (2001), proposed a model for selection of interior finishes [9]. This model handles floor coverings materials made of wood, stone, ceramic, metal, concrete, plastic, carpet, partition wall, plastering, and paintings. The factors consider in this program are a strength, durability, maintenance, ergonomics, and aesthetics and so forth. The user of the system established the weight of importance for each factor, and the system integrates this input with knowledge and proposes some material with the highest score.

d) Halil \& Mesut (2004) developed an automated building element selection system [10]. The system is responsible for the selection of building elements correctly from a vast number of alternatives in the design process. According to claims of this system, it is capable of choosing the right building element to avoid the serious problems concerned with the economy, construction functionality, and appearance.

e) Che-Ani et. al. (2009) presented a model of computer-based automation to determine the quality of housing [11]. The research has mapped the automated monitoring system to check the performance of low and medium cost housing. The study briefed about the transformation of empirical housing data into the integrated software to determine housing quality.

The discussion above reveals that earlier generation researcher also realized the importance of using IT-based monitoring system in the field of architectural design and they had developed the system to improve the design practice. Nevertheless, most of these systems were requiring the hardware skills to use and operate, as shown in Figure 1. These systems had a limitation in usage, and only professional were able to 
reap benefit from these systems. Thus, the common people were not much benefitted from these systems. Consequently, this research considers that people of developing countries need the design monitoring system that should be usable for the majority and does not require any special skills and hardware. Therefore, this research considers developing a mobile application to evaluate the housing quality. Such a mobile application in developing world would be inexpensive, comprehensive in scope and easy to use.

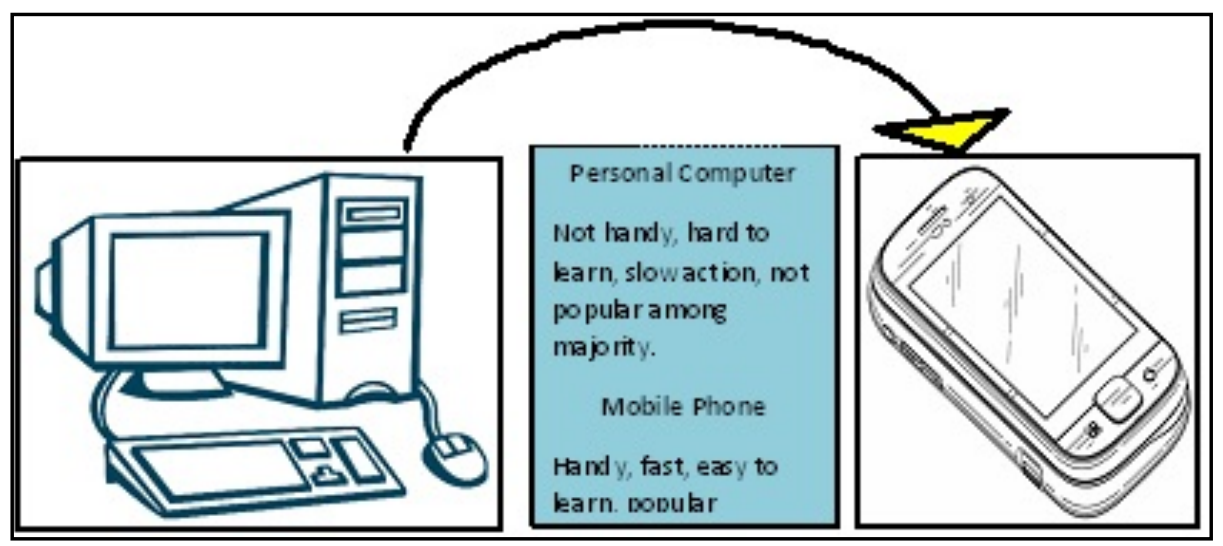

Fig. 1. $\mathrm{PC}$ vs. Mobile Phone

The mobile applications market is currently undergoing rapid expansion, as mobile platforms continue to improve in performance, and as the users' need for a wide variety of mobile applications increases [24]. According to Harrison, R et.al (2016), the usefulness of mobile devices has increased greatly in recent years allowing users to perform more tasks in a mobile context [12]. This increase in usefulness has come at the expense of the usability of these devices in some contexts. Modern day applications look very attractive and are quite easy to use, in this context Flood, et.al. (2012) added that a survey of mobile application users found that users spend on average five minutes or less learning to use a mobile application [13]. Based on available information, this research recommends that mobile application should be interactive, attractive and easy to use for most of the targeted population because the majority of people do not want to spend time on learning the application.

\section{$3 \quad$ Research methodology}

Research methodology developed in this study is resolved into three stages; StageI, II, and III. Stage-I, explains about the type of data required and its validation requirement. Stage-II, explains about various criteria of housing quality and briefly explains about subsections of quality criteria, also define the ranking of these. Moreover, stage III explores the application development mechanism. 


\subsection{Stage I - Data Collection}

This stage will work out the secondary data from study of Chohan et.al. [14], the data of housing design quality issues and quality standards capable of fulfilling the conditions mentioned in problem statement and aim of the study. The available data would be reviewed and assured that it covers all essential criteria of housing design i.e.: Site, architectural design, construction, services, maintenance, and sustainability. At this stage, it is highly desirable that available data should be a published data in reputable journals or research platforms; this will further confirm its reliability. Also, informal interviews and discussions will be conducted with professional personnel such as; architects, engineers, building maintenance contractors, project managers, and designers to endorse the reliability of available data and quality issues of housing. At this stage, it is suggested that housing quality data published by lead research will be used for development of housing quality check mobile application [14].

The study has been conducted to determine the design quality indicators and parameters for affordable housing in Karachi Pakistan [14]. The study identified that absence of quality in Karachi housing resulted from various factors ranging from policy failure, violation of bylaws, population, housing scarcity and non-availability of quality parameters, etc. Because of this trend, the end users experience the nuisance of unplanned maintenance and bear the tax of heavy repair and reworks. Study has eventually evaluated forty-eight (48) quality indicators for housing design (QIHD) farmed in seven sections [14]. The study concludes that existing design quality of affordable housing in Karachi could be enhanced through improving the design, construction, services, site development and neighborhood and sustainability, as shown in Figure 2.

\section{MODEL OF QUAUTY INDICATORS FOR AFFORDABIE HOUSING DESIGN IN}



Fig. 2. Models of Quality Indicators for Affordable Housing Design in Karachi Pakistan (Chonan et al., 2015) 


\subsection{Stage II - Housing Quality Data Flow}

At this stage, available data will be reviewed in the context of quality indicators for housing design and classified under various segments of housing design components as listed in stage-I. This practice will identify the indicators to be used as a tool to evaluate the housing design quality, and housing typology will be correlated in the context of quality indicators. The numerical scale will be set to measure the response for each quality indicator. At this stage, researchers will work in close collaboration with the software (mobile application) developer to map the entity relation and to prepare housing quality database. Stage II is shown in Figure 3.

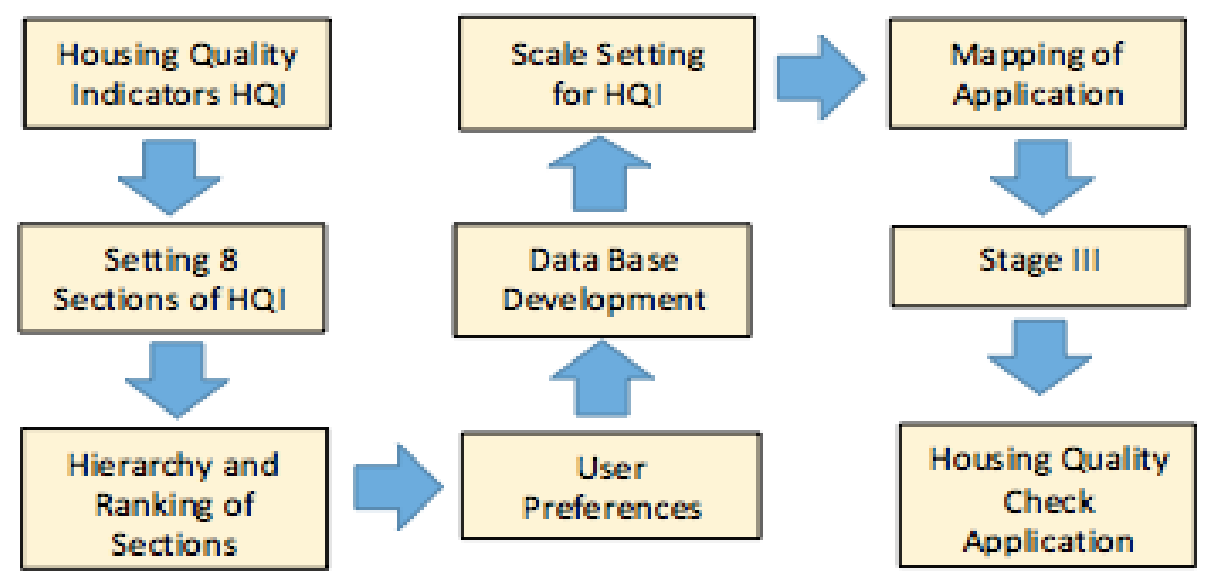

Fig. 3. Stage II (Housing Quality Data Flow)

\subsection{Stage III - Mobile Application Development}

Development of Mobile Application is the process by which applications are developed for mobile devices. Though the mobile application development process is similar to software engineering, it presents some additional requirements for which the traditional software development and process has to be customized $[15,16]$. According to the studies Dehlinger et.al and Flora et.al., hardware, software and communication are three key characteristics and features that differentiate a mobile version from a conventional desktop application $[17,18]$.

A mobile application development involves the systematic development of the application and ensures significant results for mobile developers and users. However the principal advantage of a native app (mobile app) is that it optimizes the user experience; the app will operate more quickly because it's been designed specifically for particular platform [26] .

Furthermore, it becomes a reason that mobile applications do not set any prerequisite for its usage, except the condition of operating system in some cases. According to Abrahamsson et. al., software development for mobile platforms comes with unique features and its development environment and the technologies that support 
the software are different compared to "traditional" settings [19]. The study further revealed that there are nine main elements involved in the development phase such as phasing and placing, architecture line, mobile test-driven, development, continuous integration, pair programming, metrics, agile software process, off-site customer and user-centered focus.

To accomplish the aim of the research, the researcher has customized the application development stages in the domain of housing quality and its assessment. The development stages presented in this study are based on "Mobile Application Development - Best Practices" model presented by Flora et.al [25]. This study applies following development steps, which demarcates various stages of application development i.e. from conceptual to completion stage. This study considers that following 07 steps of development protocols are necessary for the successful development of "housing check" mobile application. Table 1, provides a quick review of various stages proposed for development of housing quality check mobile application.

Table 1. Stage iii (quick review of housing check application development)

\begin{tabular}{|c|c|c|}
\hline Stages & Objectives & Approach \\
\hline \multirow{3}{*}{ Application Planning } & Understanding Product & $\begin{array}{l}\text { Product Summary } \\
\text { Pre-planning }\end{array}$ \\
\hline & Product Concept Sharing & $\begin{array}{l}\text { User Definition } \\
\text { Initial product analysis }\end{array}$ \\
\hline & Project Set-up & $\begin{array}{l}\text { Development process coordination } \\
\text { Project resource coordination } \\
\text { Pre study }\end{array}$ \\
\hline Application Needs & $\begin{array}{l}\text { User Need Understand- } \\
\text { ing }\end{array}$ & $\begin{array}{l}\text { Review of the user is a friendly application. } \\
\text { Coordination of various application platforms }\end{array}$ \\
\hline $\begin{array}{l}\text { Application Design \& } \\
\text { Sociability }\end{array}$ & Interface Design & $\begin{array}{l}\text { Analysis of non-functional requirement } \\
\text { Architecture definition } \\
\text { Pattern management }\end{array}$ \\
\hline Application Development & $\begin{array}{l}\text { Implementation \& Prepa- } \\
\text { ration }\end{array}$ & $\begin{array}{l}\text { Selection of popular development platform. } \\
\text { Housing quality environment setup. } \\
\text { Development planning. }\end{array}$ \\
\hline Application Testing & System Test & $\begin{array}{l}\text { Acceptance Test } \\
\text { User Test } \\
\text { Launching Test }\end{array}$ \\
\hline $\begin{array}{l}\text { Application Security \& } \\
\text { Privacy }\end{array}$ & $\begin{array}{l}\text { Security \& privacy } \\
\text { threats }\end{array}$ & Malware apps and antivirus \\
\hline Application Analysis & $\begin{array}{l}\text { Horizontal and vertical } \\
\text { growth in HQI. } \\
\text { Deletion of HQI. }\end{array}$ & $\begin{array}{l}\text { Easy maintenance and alterations of HQI at the } \\
\text { different platform of application. }\end{array}$ \\
\hline
\end{tabular}

Application Planning: To initiate with the planning a prior research is required in the context of Housing quality variables and assessment methods. The initiative will ensure that application holds the value for users, could bring extra feature, utility, and productivity. For this purpose, a proper plan should be designed with a specified set of actions, thus allowing the efficient strategy to attract the potential users. During the planning process, data received from stage I, housing quality database (HQDB) will 
be placed in a cross-platform environment, and housing quality variables will be defined, set in the hierarchy and ranked, simultaneously other multiple tasks will be in progress such as entity relation, application interface, etc.

Application needs: After the planning process, housing quality planning and logic will be discussed at this stage. Data gathered from stages I and II will be documented, and housing quality criteria will be interrelated with the type of application users and housing typology. This is the most critical phase of the entire mobile software development life cycle. At this stage, it would be decided to empower users by allowing them to choose and control the way their data is collected and used, by maintaining the simplicity and clarity of the application. The planning strategy will ensure the better user interaction, performance, and limited resource utilization, followed by frequent and rapid iterations of requirements reviews to have respect for context.

Application design and sociability: At this stage of all design and interface of application will be determined as per the aspiration of the client (researchers) and users. However, a simple design without consuming too many resources creates a simply suitable architecture for the mobile applications. Whereas, the aspect of functionality in mobile application will remain consistent across platforms, at the same time as meeting the requirements of each platform's appearance and impression. Horizontal and vertical development in housing quality variables will also consider at this stage and type of application frameworks will use that allow the application to be easily modified and reconfigured while in the hands of users. Regarding sociability and friendliness, it has been discussed in the literature review (section 1 and 2) that contemporary users do not spend more than five minutes to learn and use the application. This trend suggests that application should be most useful text and graphics that are globally recognizable as well as culturally sensitive. The application design must present easy access and navigation, making users feel comfortable by providing fastloading content and intuitive buttons without any tutorial or additional help.

Application Development: At this stage, the lead researcher and application developer will work in close collaboration to ensure that application requirements are being addressed perfectly. The developers will count on every single mobile platform and ergonomics. Whereas, the lead researcher will ensure that there should be crossplatform development as much as possible by creating reusable codes to speed up application development. Both application developer and lead researcher will try to have some features light for the first version of the application to develop for a single platform and later port on the other platforms.

Application Testing: Application-testing will be carried on real devices, with real users. This will determine the levels of usability and power consumption to verify that application is not a battery guzzler. Testing will be should frequently be done, iteratively, early and as often as possible. Also, it should also be tested on multiple browsers, devices, on a variety of platforms for cross-platform compatibility with different screen sizes and different carrier connections, network to confirm that they accommodate both online and offline usage.

Application Security \& Privacy: Wireless transmission in mobile devices is often susceptible to attack by unauthorized users as they download and upload data wirelessly, in potentially insecure locations. It is common belief that belief that mobile 
application does not have integrated platforms and inherent security capabilities and some encryption of sensitive data across open or unsecured networks is possible. Therefore application will be developed with security features such malware apps and antivirus. Also, to it after the testing, the application will be registered so that any future legal problems may be avoided.

However, for privacy issues, this application will introduce a policy regarding use of components across platforms, which facilitates maintenance and extensibility across applications on different platforms. The policy will demarcate; practice privacy by design, communicate openly \& efficiently, use clear and straightforward language, make privacy policy easily accessible, use enhanced notice, provide users with choices \& controls, empower users, secure users data and ensure accountability.

Analysis of Application: Bring an element of continuous improvement in the application. This study is proposing to monitor the performance of app in the market monitored by an analyst. This will help to work out the additional requirements of users that could be further added as a new feature for a later version. This analysis will also highlight the features that are not used, and those can either be removed or made easier to access in future. Combining this response will endorse which features or capabilities are mostly used by the user and eventually lessen the technical support issues. This study considers that this practice will result in improvements and will provide insight for future application updates.

\section{$4 \quad$ Model of housing quality check mobile application}

The development of this application will provide the unique opportunity to users to evaluate the quality of existing/proposed housing through their mobile device. Consequently, user of this application will feel self-reliance in selecting the property and would not deem to believe in ivy words of property vendors. Figure 4, shows the model of housing check mobile application. The application will start with welcome note and a simple definition of housing quality. At this moment, users will be guided in candid manner to get familiar with environment of application. After this brief introduction and tutorial, application will switch to other platform and inquire about type of housing (typology), user intends to evaluate. Application will convey this information to next platform (QIHD) and application will list up the series of question set to evaluate housing quality. User will rank the various quality indicators with given scale and response of users will be conveyed to next platform (analysis). Application will analyze the response and covey the results to final platform (output). User will learn the evaluation results in term of average housing, good hosing and excellent housing. Figure 4, shows the model of housing check mobile application. 


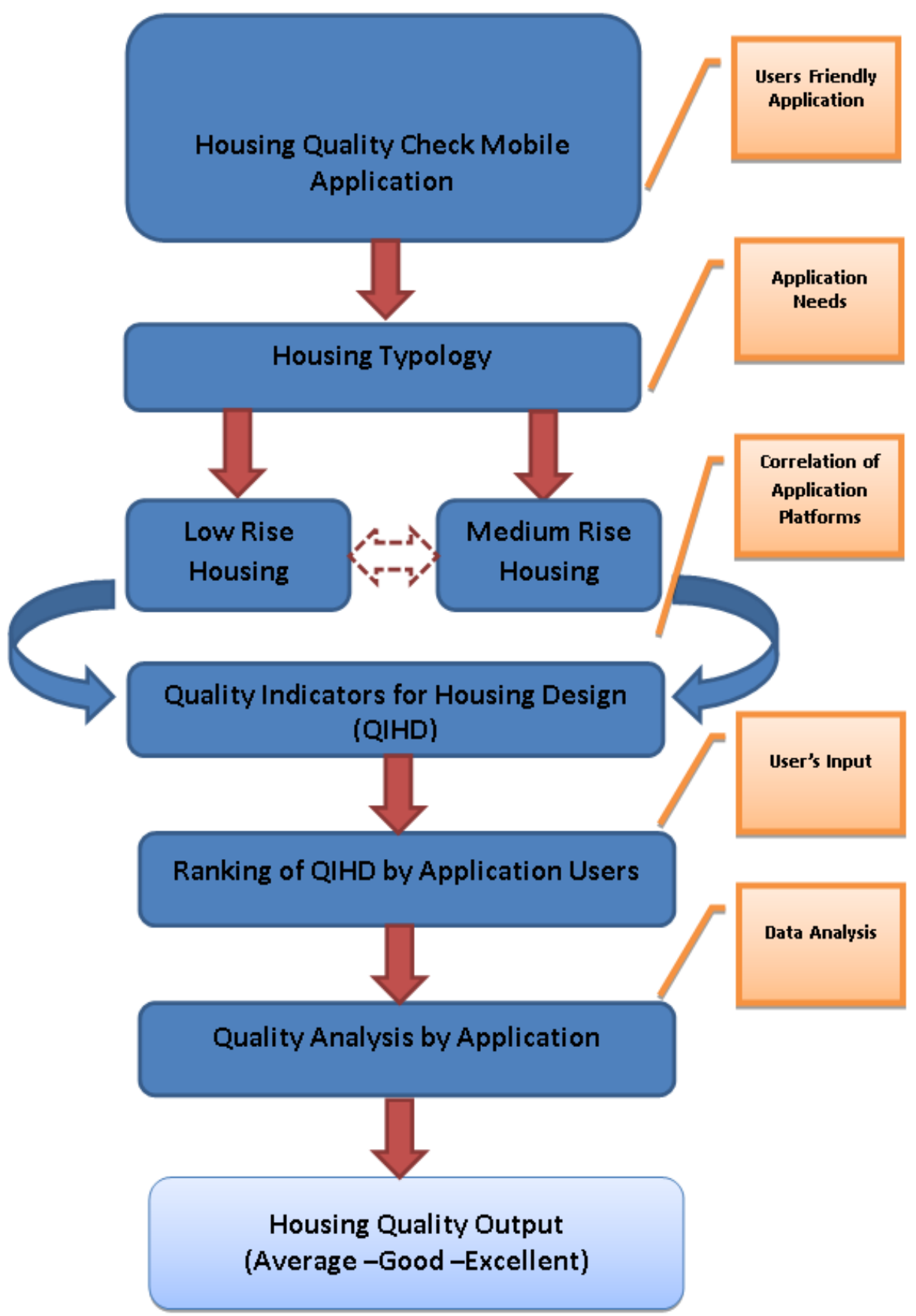

Fig. 4. Model of Housing Quality Check Mobile Application 


\section{$5 \quad$ Contribution to field}

The proposed outcomes of this study (Mobile Application) hold multi-dimensional benefits for ordinary people as well as professionals involved in housing design and retail such as:

a) To facilitate the common person to work out the quality of housing and best option for his investment in housing.

b) Provide opportunity to professional surveyors to conduct housing surveys.

c) Tool for design professional to learn the trends of design preference.

d) Tool for evaluators to ascertain the condition of housing for various purposes, e.g. mortgage, dilapidation, safety, insurance, etc.

\section{Conclusions}

During last decade, mobile device users have increased drastically and resulted in rapid growth in allied industrial of mobile devices. The quick development of this new computing platform has almost outclassed conventional software engineering processes to personalized mobile application development. Consequently, the variety and availability of mobile applications are increasing rapidly. Furthermore, the increased processing power of portable devices provides an edge to developers of developing various types of apps to serve a range of services. Many sectors are successfully using the mobile application and promoting their business, such as traveling and tourism, motor vehicle registration, and renewal, toll tax, police, government taxation and revenue collection, urban service providers are widely using the mobile application to facilitate their customers.

However, there is still an absence of novel research initiatives around the mobile application development process in housing and real estate sector. Therefore, this research has mapped the plan to develop the mobile application for potential owner or renter to evaluate the housing quality for their prospect house. This application will help the users to ascertain the housing quality through various parameters such as site, architectural design, construction, services, etc. Though, there are certain issues for users of the mobile application in the overall i.e. small size of mobile devices, limitation of interaction, small screen size, poor connectivity and limited input modalities have an effect on the usability of mobile applications. Despite these issues, the mobile application is shaping the future of services and helping the people to save their precious time, resources and energy.

\section{$7 \quad$ Acknowledgement}

The authors would like to express our heartfelt thanks to Ajman University of Science and Technology, UAE and Universiti Kebangsaan Malaysia, MALAYSIA for supporting this research. Credit also goes to various organizations, which facilitated the successful completion of this research. 


\section{$8 \quad$ References}

[1] United Nations (2014). Revision World Urbanisation Prospectus 2014.

[2] UN-Habitat, 2014. World Development Indicators, World Bank, Washington DC 2014

[3] UN-Habitat 2016. URBANIZATION AND DEVELOPMENT Emerging Futures. World Cities Report 2016, United Nations Human Settlements Programme (UN-Habitat) 2016.

[4] University of Twente (2009) Social Domain Tenure Model, International Institute for GeoInformation Science and Earth Observation (ITC) 2009.

[5] Cyber Gear. (2016). Why Mobile Apps Are Important For Your Business [Online]. Available: http://www.cyber-gear.com/main/mobile-apps.php (visited July 14, 2016).

[6] S. Shane. (2016). Why Your Small Business Needs a Mobile App [Online]. Available: https://www.entrepreneur.com/article/269978 (Visited; 14 July 2016)

[7] P. Jackson, Introduction to Expert System. England: Addison-Wesley, 1999

[8] H. Rivard, C. Bedard, and P. Fazio, "Shaped conceptual model for building envelope design process", Building and Environment, vol. 34, 1999.

[9] C.A. Altunay, A knowledge-based system for alternative selection of internal finishes. Gazi Magusa, Mersin, Turkey: Eastern Mediterranean University, 2001.

[10] Z. Halil and B. Mesut, "A Building Elements Selection System for Architects", Building and Environment, vol. 39, pp. 307 - 316, 2004. https://doi.org/10.1016/j.buildenv.2003. $\underline{09.010}$

[11] A.I. Che-Ani, A.H. Chohan, N.A.G. Abdullah, M.M. Tahir, M.F.M. Zain, and N. MohdTawil, "The Potential to Develop Design Quality Automation for Housing”, World Academy of Science, Engineering and Technology, vol. 29. 2009

[12] R. Harrison, D. Flood, and D. Duce, Usability of Mobile Applications: Literature Review and Rationale for a New Usability Model. Oxford Brookes University, 2016.

[13] D. Flood, R. Harrison, C. Iacob, and D. Duce, "Evaluating Mobile Applications: A Spreadsheet Case Study", International Journal of Mobile Human-Computer Interaction, vol. 4(4). 2012 https://doi.org/10.4018/jmhci.2012100103

[14] A.H. Chohan, A.I. Che Ani, and J. Awad, "Development of Quality Indicators of Housing Design (QiHD)", Open House International, vol.40, no.4, December 2015.

[15] L. Williamson, "A mobile application development primer. A guide for enterprise teams working on mobile application projects". IBM Whitepaper. 2012.

[16] A. Wasserman, "Software engineering issues for mobile application development," Proceedings of the FSE-SDP workshop on Future of software engineering research - FoSER, 2010, pp. 397-400.

[17] J. Dehlinger, and J. Dixon, "Mobile Application Software Engineering: Challenges and Research Directions." Workshop paper. Oct 2011.

[18] H.K. Flora, X. Wang, and S. Chande, "An Investigation of Characteristics of Mobile Applications: A Survey Study," International Journal of Modern Education and Computer Science, vol.6, no. 6, 2014.

[19] P. Abrahamsson, A. Hanhineva, H. Hulkko, T. Ihme, J. Jäälinoja, and M. Korkala, "Mobile-D: an agile approach for mobile application development". Conference on Object Oriented Programming Systems Languages and Application; Companion to the 19th annual ACM SIGPLAN conference on Object-oriented programming systems, languages, and applications Vancouver: ACM, 2004, pp. 174-175.

[20] CABE .2005. Housing audit Assessing the design quality of new homes in the North East, North West and Yorkshire \& Humber. The Commission for Architecture and the Built Environment. England 
[21] UN-Habitat. URBANIZATION AND DEVELOPMENT Emerging Futures. World Cities Report 2016, United Nations Human Settlements Programme (UN-Habitat) 2016.

[22] UN-Habitat. World Development Indicators, World Bank, Washington DC. 2014

[23] United Nations. Revision World Urbanisation Prospectus. Washington DC. 2014.

[24] Spataru, A.C. Agile Development Methods for Mobile Applications, Master of Science Computer Science School of Informatics University of Edinburgh.2010

[25] H.K. Flora, X. Wang, and S. Chande An Investigation into Mobile Application Development Processes: Challenges and Best Practices. I.J. Modern Education and Computer Science, 2014, 6, 1-9 https://doi.org/10.5815/ijmecs.2014.06.01

[26] Wright, N. Native Mobile Application. https://www.upwork.com/blog/2014/02/choosingbest-mobile-app-development-technology/.CEO at AppInstruct. (29.4.2017)

\section{Authors}

Afaq Hyder Chohan is with the Department of Architectural Engineering, College of Engineering, Ajman University of Science and Technology, Ajman, UAE (afaqhyder@yahoo.com).

Haryanti Mohd Affandi is with the Department of Teaching and Learning Innovation, Faculty of Education, Universiti Kebangsaan Malaysia, Bangi, Selangor, MALAYSIA (haryantima@ukm.edu.my).

Jihad Awad is with the Department of Architectural Engineering, College of Engineering, Ajman University of Science and Technology, Ajman, UAE (dr_jihadaa@yahoo.com).

Adi Irfan Che-Ani is with the Department of Architecture, Faculty of Engineering and Built Environment, Universiti Kebangsaan Malaysia, Bangi, Selangor, MALAYSIA (adiirfan@gmail.com).

Article submitted 31 October 2016. Published as resubmitted by the authors 23 July 2017. 